\title{
The MYCN Oncogene
}

\author{
Leanna Cheung, Jayne E. Murray, Michelle Haber and Murray D. Norris
}

Additional information is available at the end of the chapter

http://dx.doi.org/10.5772/54813

\section{Introduction}

$M Y C N$ is a member of the MYC family of oncogenes, which also includes $c-M Y C$ and MYCL. Despite knowing about the existence of MYCN for nearly thirty years, the majority of functional studies involving MYC family members have focused on c-MYC due to the limited expression profile of MYCN in human cancers, and also in part due to the existence of highly conserved functional domains between c-MYC and MYCN [1]. MYCN is normally expressed during embryonal development and orchestrates cell proliferation and differentiation in the developing peripheral neural crest [2]. However, the deregulated expression of MYCN has been shown to contribute to tumorigenesis and neuronal transformation [3]. Thus, MYCN represents a highly desirable therapeutic target. Previous studies have shown that downregulating MYCN expression, via antisense oligonucleotides, resulted in lower tumour incidence and decreased tumour mass in a murine neuroblastoma tumour model [4]. However, to date, no molecularly targeted therapies have been developed that are able to mimic this response in the clinic, and further studies are required to help elucidate the mechanisms that drive MYCN tumour formation and progression.

\section{The MYC family and the discovery of MYCN}

The eventual discovery of MYC oncogenes arose from early pioneering work on the Rous sarcoma virus (RSV), a transforming retrovirus able to cause sarcomas in infected chicken cells. Using the information provided by RSV, hybridisation studies were performed on a specific group of avian tumours involving a retrovirus responsible for inducing myeloid leukaemia. This led to the identification of a sequence that was named $v$-gag-myc, or $v-m y c$ for myelocytomatosis (the leukaemia that is induced following the transduction of avian cells with this virus) and supported the idea that viral integration into a host genome could activate a nearby host oncogene [5,6]. As it transpired, the human homologue of $v$-myc, termed $c-M Y C$ (cellular-MYC) was the first cellular oncogene whose overexpression was 
shown to be activated through retroviral insertional mutagenesis [7]. Deregulated expression of $c-M Y C$ has since been implicated in a range of cancers, and allowed the discovery of other important MYC family members including MYCN and MYCL.

Neuroblastoma is the most common extracranial solid tumour of early childhood and accounts for approximately $15 \%$ of all cancer related deaths in children. Aggressive drug refractory neuroblastoma cells have been frequently observed to contain genomic aberrations referred to as double-minute chromatin bodies and homogeneously staining regions. Both of these types of aberrations were found to contain multiple copies or amplification of specific genes, and in particular, the critical gene within these regions was later identified to be the $c-M Y C$-related oncogene, $M Y C N$, so-called because of its identification in neuroblastoma cells [8]. Amplification of the $M Y C N$ oncogene has also been demonstrated in retinoblastoma, glioblastoma, medulloblastoma, astrocytoma and small cell lung cancer cells [9]. In addition, another member of the MYC-oncogene family, MYCL, was identified in small cell lung cancer (SCLC), and demonstrated homology to a small region of both $c-M Y C$ and $M Y C N$. Gene mapping studies assigned MYCL to human chromosome region 1p32, a location that is distinct from that of either $c-M Y C$ or MYCN (regions 8q24 and 2 p24 respectively) but is also associated with cytogenetic abnormalities in certain human tumours such as thyroid cancer and lung cancer [10,11]. MYCL was found to be amplified in some SCLC cells [12]. In mammals, a fourth member of the MYC family, s-Myc has been identified, however only $c-M Y C, M Y C N$ and $M Y C L$ have been implicated in the tumorigenesis of specific human cancers [13].

All three tumour-associated MYC genes have the same characteristic three-exon structure with the major polypeptide open reading frame residing in the second and third exons. The first exon is not conserved between the genes, but rather possesses regulatory functions, whereas the two coding exons produce highly homologous sections of amino acids interspersed with areas of diminished conservation, leading to the suggestion that individual MYC polypeptides have discrete, independent, functional domains [14]. In tumour biology, many cancers have been shown to exhibit increased levels of MYC protein in tumour tissue relative to the surrounding normal tissues, and this has been shown to contribute to the aggressiveness of the tumour [15]. Importantly, the MYC family of proteins share functionally similar roles, acting as transcription factors to drive cellular proliferation and vasculogenesis, promote metastasis and genomic instability, as well as inhibit cell differentiation and reduce cell adhesion $[13,16]$. However, recent findings have also raised the possibility of transcriptionally-independent functions of the MYC proteins [17].

\section{The functional activity of MYCN}

MYC proteins are well established as nuclear phosphoproteins that act as regulators of transcription, and can both activate and repress the expression of its target genes [16]. MYCN encodes a $60 \mathrm{kDa}$ protein that has affinity for and binds to DNA, and is phosphorylated by casein kinase II $[18,19]$. Phosphorylation is important for the transforming abilities of MYC family members and also for the regulation of MYC protein stability and activity [20]. The 
affinity of MYCN protein for DNA relies on the presence of certain motifs, comprising a basic DNA binding region, an $\alpha$-helical protein-protein interaction domain or helix-loop-helix $(\mathrm{HLH})$, and a leucine zipper motif (Zip) encompassing the bHLH-Zip domain at the carboxy or C-terminus of the protein [9]. The mechanism that mediates the DNA-binding capacity of MYC proteins was confirmed via the identification of MAX, also a bHLH-Zip protein [21]. MYCN and MAX (Figure 1) interact to form a complex that binds to DNA in a sequence specific manner [22]. MYCN binds to MAX protein via its bHLH-LZ region. Several other proteins have also been shown to interact with the C-terminus of MYCN, including YY-1, AP2, TFII-I and BRCA1 [23], or with the central region of MYCN such as NMi [24], all of which are associated with MYCN's function as a transcriptional regulator.

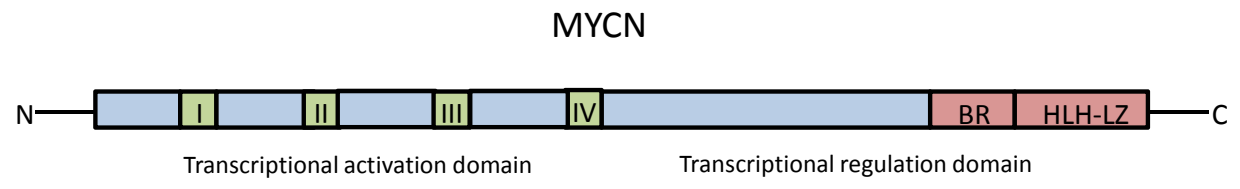

MAX

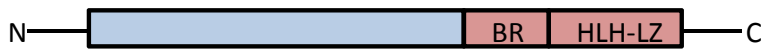

Figure 1. Domains of the MYCN and MAX proteins. The N-terminus of MYCN has three elements, known as MYC homology boxes I-III, which are highly conserved in MYC proteins. The C-terminus contains the basic-region/helix-loop-helix/leucine-zipper that is responsible for interaction with the MAX protein.

The amino or N-terminus of MYCN acts as a transactivation domain that contains two highly conserved regions called Myc Homology Boxes I and II (MBI and MBII) [1]. This region has been shown to bind to nuclear cofactors, including TRRAP, p107, BIN1, MM-1, AMY-1, PAM, $\alpha$-Tubulin, TIP48 and TIP49, to assist the targeting of protein to specific gene promoters [23, 25]. Another protein YAF2, has been demonstrated to bind to the central region of MYCN to further stimulate transcription upon MYCN-MAX transactivation [26]. All of these interacting proteins are a part of a transcription factor complex by which target genes are activated. Myc Homology Box III (MBIII) is conserved only within c-MYC and MYCN, but not MYCL, and is necessary for cellular transformation [27]. A fourth Myc Homology Box (MBIV) is also necessary for MYC transforming activity [28].

Recent studies have provided evidence of a function of MYCN that is independent from its role as a classical transcription factor. MYCN was shown to remodel large domains of euchromatin, regions of lightly packaged chromatin that contain active, functioning genes, by regulating histone acetylation $[29,30]$. Two possibilities have been suggested for this role. The first is that MYCN maintains the activity of euchromatin, whilst the second is that MYCN maintains euchromatin at remote sites to act as an enhancer and regulator of genes at a distance. Novel functions of other MYC proteins have been identified through mutational analyses that have uncoupled the transforming ability of c-MYC from its role as 
a transcription factor [28, 31]. c-MYC was found to increase the translation of specific mRNAs by promoting the methylation of the 5 ' mRNA guanine "cap", including mRNAs encoding cyclin T1 and CDK9 [31]. A role for c-MYC has also been described in the initiation of DNA replication by binding to various components of the pre-replicative complex and localising to early sites of DNA replication [32]. These observations suggest that c-MYC may play a role in controlling initiation of the $S$ phase of the cell cycle and contribute to replicative stress and genomic instability, to further accelerate tumorigenesis [17]. Even though the evidence has yet to be provided, given the high level of homology between cMYC and MYCN, the described transcription-independent roles of c-MYC suggest similar roles will be identified for MYCN in contributing to tumour cell biology.

\section{MYCN as a transcriptional activator}

As indicated above, MYCN heterodimerises with MAX and binds with high affinity to a CACA/GTG E-box sequence found upstream of promoter target sequences [13]. The MYCNMAX heterodimer activates transcription via several mechanisms. TRRAP (or TRansactivation/tRansformation Associated Protein) binds to the N-terminal region of MYCN and is essential for MYCN transformation. Through TRRAP, MYCN recruits histone acetylation (HAT) complexes to chromatin, including the 1.8 megaDalton SAGA complex (SPT/ADA/GCN5/Acetyltransferase) [33]. Histone acetylation is associated with gene activation by chromatin modification influencing histone-DNA and histone-histone contact [34]. TRRAP is involved with another HAT complex, TIP60, an $\mathrm{H} 2 \mathrm{~A} / \mathrm{H} 4$ acetylase [35]. Interestingly, in vivo acetylation of histone $\mathrm{H} 4$ is highly associated with MYC target gene activation [36]. Two other proteins, TIP48 and TIP49 that are found in the TIP60 complex also bind to the N-terminus of MYCN [25]. Both proteins are highly conserved hexameric ATPases that are involved in chromatin remodelling involving the movement or displacement of nucleosomes, as opposed to chromatin modification [37].

The MYC family represents a particularly unusual set of transcription factors in that they can bind to and regulate approximately $10-15 \%$ of the entire genome [14]. Some MYCN target genes have been shown to be activated independently of TRRAP and HAT complexes. Investigation into HAT independent activation has revealed the involvement of RNA polymerase II at the promoter regions of target genes. c-MYC protein binding has been shown to stimulate the clearance of RNA polymerase II from the promoter region to allow for efficient transcription elongation by the RNA pol II kinases, TFIIH and positive transcription elongation factor b (PTEFb) [38]. c-MYC also regulates RNA pol II promoter clearance by controlling the expression of RNA pol II kinases via mRNA cap methylation, polysome loading, and the rate of translation [31].

\section{MYCN as a transcriptional repressor}

Most studies have focused on the role of MYC proteins as transcriptional activators. However, cells transformed by constitutive expression of c-MYC are characterised by the loss of expression of numerous genes such as those involved in cell adhesion and cell cycle regulation, and even loss of $c-M Y C$ itself [39-41]. An early indicator of the transcriptional 
repressor role of MYC proteins was the involvement of c-MYC in a negative feedback loop, where the introduction of ectopic c-MYC or MYCN was able to downregulate endogenous expression of c-MYC in mouse fibroblast cells [42]. Structure and function analyses found that the regions of $\mathrm{c}-\mathrm{MYC}$ that are required for transformation are also required for negative autoregulation [43] and led to the idea that the repression of target genes by MYC proteins could also contribute to transformation.

The understanding of transcriptional repression by MYC proteins was greatly advanced via the identification of repressed target genes such as transglutaminase-2 (TG2) and interleukin-6 (IL-6) [44]. Genomic studies have now revealed that MYC proteins repress as many targets as they activate, emphasising the role of gene repression by these oncoproteins during cellular transformation [14]. One recent example is the identification of TG2 repression by MYCN in neuroblastoma, which occurs via the interaction between MYCN with Specificity Protein I (SP1) [45]. TG2 is a multifunctional enzyme that catalyses the transamidation and multimerisation of proteins, but also promotes programmed cell death and induces neuritic differentiation in neuroblastoma cells [46]. Hence downregulation of TG2 by MYCN would allow neuroblastoma cells to overcome apoptosis and continue to proliferate. Similarly, MYCN has been shown to interact with SP1 to downregulate the expression of MRP3 (also known as $A B C C 3$ ), the gene encoding an intermembrane transporter which is involved in the transport of organic anions, prostaglandins, leukotrienes and selected chemotherapeutics [47-49]. Another important gene that is downregulated by MYCN is IL-6, which has been shown to play an important anti-angiogenic role by inhibiting vascular endothelial cell proliferation [50]. The transcriptional repression by MYCN is also supported by the interaction between MYC proteins and another transcription factor MIZ-1 (Mycinteracting zinc finger protein-1) [51]. MIZ-1 is a POZ/BTB (poxvirus and zinc finger/bric-abrac, tramtrack and broad complex) domain protein that transactivates genes involved in cell cycle regulation as well as tumour suppressor genes via the recruitment of the p300 histone acetyltransferase [52]. Interestingly, high-level MIZ-1 expression is associated with a favourable disease outcome of neuroblastoma [53]. MIZ-1 interacts with the carboxyterminal HLH region of c-MYC and MYCN, where the binding of the MYC-MAX heterodimer to MIZ-1 disrupts the interaction between MIZ-1 and p300, causing the transcriptional repression of tumour suppressor genes [54]. MYC has also been shown to recruit a DNA methyltransferase, DNMT3a to the MYC-MIZ-1 complex, suggesting that repression can be mediated by the methylation of target gene promoters [55].

\section{Mechanisms of regulating MYCN expression}

Due to the gross transforming ability of deregulated expression of MYC proteins, the expression of these protooncogenes is tightly regulated in normal cells at both the transcriptional and protein level. For example, MYC mRNA transcripts and proteins have very short half-lives and are expressed at constant levels as cells enter the cell cycle [56, 57]. Furthermore, anti-proliferative signals trigger rapid down-regulation in expression, and the phosphorylation patterns of MYC proteins are known to influence their stability. In addition to these mechanisms, expression of $\mathrm{MYCN}$ is particularly tightly regulated with regards to 
timing and tissue specificity. Thus, MYCN is normally expressed during embryonal development of the peripheral nervous system in neural crest cells [2]. Neural crest cells migrate during mid-gestation to populate the entire peripheral nervous system, including autonomic and peripheral ganglia and the adrenal gland. These migrating progenitor cells represent a highly proliferative population, and during normal development exit the cellcycle and undergo differentiation following the colonisation to the ganglia and spinal cord area. This event is orchestrated by extracellular signalling molecules such as mitogens and cytokines and coincides with decreased expression of MYCN [56, 58]. Without this strict control, dysregulated MYCN expression impairs the ability of progenitor cells to undergo differentiation. Studies which sustained MYCN expression in murine neural crest cells under the control of a tyrosine hydroxylase promoter, demonstrated the capacity to cause neuroblastoma in transgenic mice [3]. Despite this transforming ability, MYCN is vital for normal embryonic development, and murine embryos lacking MYCN exhibit profound hypoplasia, particularly in the central and peripheral nervous system, disorganized architecture of the brain, defective heart development and defects in the lung, genitourinary system, stomach, intestines and limb buds [59].

In order to understand how extracellular stimuli controlled MYC expression in cells, gene mapping studies in association with MYC transcription studies were undertaken, and these identified response elements within the MYC transcript as well as their regulators. In neuronal cells, MYCN has been shown to be regulated in its promoter region as well as in an enhancer region upstream of the coding region. The elongation transcription factor, E2F binds to the promoter region of MYCN in response to different mitogenic signals [60]. The promoter region also contains positive transcription factor binding sites for SP1, SP3 and TGF $\beta$ [61]. However, the presence of a retinoic acid response element (RARE) within this region allows for negative regulation of $M Y C N$ by retinoic acid [62].

A key finding was made in 1986 which identified $c-M Y C$ as the first eukaryotic gene to be negatively regulated by transcriptional elongation control, where a block in the elongation of mRNA during transcription occurred during cellular differentiation [63]. This finding was later confirmed in $M Y C N$ studies where transcription elongation pausing sites were identified in exon 1 and intron 1 of human $M Y C N$ [64, 65]. Furthermore, there is in vivo evidence that the downregulation of $M Y C N$ during mouse embryogenesis is partly regulated by the control of transcriptional elongation [66].

Transcription alone cannot account for the large difference in mRNA levels following the introduction of proliferative or anti-proliferative stimuli. The rapid turnover of mRNA was also associated with the discovery of two distinct mechanisms of MYC mRNA decay. The first involves a translation-independent mechanism involving poly(A) tail shortening of the untranslated region of the transcript, while the second represents a translation-dependent mechanism that is regulated by a region of mRNA which corresponds to the C-terminus of the protein, called the coding region determinant [67-69]. This region is bound to a $75 \mathrm{kDa}$ protein that protects the region of mRNA from endonuclease attack, in response to growth signals that induce c-MYC stabilisation. In the case of MYCN, RNA stability factors have also been identified which bind to the untranslated region of MYCN mRNA. In addition, an 
internal ribosomal entry segment (IRES) in the transcript acts to enhance neuronal specific translation [70, 71].

\section{Regulation of MYCN protein expression}

The regulation of MYCN protein levels has also been investigated and phosphopeptide analysis has revealed that specific serine and threonine residues of MYCN are phosphorylated in vivo. Two residues in particular, Threonine 58 (Thr58) and Serine 62 (Ser62) have been demonstrated as important determinants of transformation and MYCN protein stability and activity [20]. Proliferative stimuli activate phosphorylation of Ser62 by cyclin B and Cdk1 during prophase to increase MYCN protein stability [72]. Phospho-ser62 via a feedback mechanism, then serves as a platform for the phosphorylation of Thr58 by glycogen synthase kinase 3 (GSK3), allowing the tumour suppressor FBW7 to bind and recruit a ubiquitylation complex, directing $\mathrm{MYCN}$ protein for degradation. Mitotic degradation of MYCN in the absence of growth factor-dependent signals allows cell cycle exit and the commencement of differentiation [73]. Another kinase, Aurora A, has recently been identified and shown to inhibit degradation of ubiquitinated MYCN by supporting the synthesis of non-degradable ubiquitin chains [74].

\section{MYCN downstream target genes}

The first transcriptional target for a MYC protein was discovered ten years after the identification of human $c-M Y C$. The development of a conditionally expressed $c-M Y C$ construct, via the fusion of human $c-M Y C$ to the hormone-binding domain of the oestrogen receptor, led to the identification of a downstream target involved in cell cycle progression, $\alpha$-prothymosin [75]. This approach was then used to identify additional targets including ornithine decarboxylase 1 (ODC1), the rate-limiting enzyme involved in polyamine synthesis [76]. A different method of identifying MYC targets utilised MYC-null models to determine whether the regulation of expression of genes was dependent on the presence of a MYC oncogene. Such examples of labour-intensive techniques were invaluable in determining single bona fide MYC targets, however recent advances in technology have allowed for large-scale analyses of MYC-regulated genes [77, 78].

Expression microarrays and chromatin immunoprecipitation assays (ChIP) have helped researchers identify MYC-regulated targets as well as link MYC-target expression to functional cellular pathways which are associated with transformation [79, 80]. MYC and MYCN-regulated targets have since been linked to a number of transforming activities involving the cell cycle (eg. cyclin D2, CDK4, p21), cell proliferation (e.g. MDM2), growth, metabolism (e.g. ribosomal proteins, proteins involved in nucleotide biosynthesis such as thymidylate synthase and ODC1), cell adhesion and migration (e.g. integrins) and angiogenesis (e.g. thrombospondin) [81-86]. Indeed, the activation and repression of MYC target genes is a well-coordinated event. Time course studies using microarray have identified differences between early and delayed gene expression responses, following MYC activation in a MYC-inducible cell system [87]. Early-response MYC target genes are 
primarily involved in MAPK signalling, RNA metabolism and transcription factors, which suggests a program that prepares cells for entry into the $S$ phase. On the other hand, delayed-response MYC target genes are involved in ribosomal biogenesis, nucleotide metabolism and energy metabolism, suggesting subsequent maintenance of cells during the $S$ phase. Finally, late steady-state MYC-mediated transcription involved genes that regulate the cell cycle, nucleotide metabolism and DNA replication. Most genes that were activated in the early response were then repressed during this late steady-state phase. Furthermore, sustained MYC activation led to the silencing of differentiation-related genes and upregulation of genes that are involved cell proliferation.

During tumorigenesis, MYCN promotes cell cycle progression by the activation of cyclins (such as cyclin D1 and D2) as well as cyclin-dependent kinase 4 (CDK4), and represses the expression of mediators of cell cycle arrest such as p21 [73]. One important MYCNregulated metabolic pathway involves the synthesis of polyamines, which are organic cations that enhance transcription, translation and replication [88]. MYCN expression is strongly correlated with $O D C 1$ expression in neuroblastoma, and the high levels of ODC1 expression that are driven by $M Y C N$-amplification and over-expression are strongly associated with poor clinical outcome of this disease [89].

Another gene whose expression is strongly correlated with MYCN expression in neuroblastoma is that encoding the multidrug resistance-associated protein, MRP1, a glycoprotein that belongs to the superfamily of ATP-binding cassette (ABC) transmembrane transporters [90-92]. MRP1, also known as ABCC1, is able to confer resistance to a broad range of structurally unrelated chemotherapeutic drugs [93]. MRP1 has since been shown to be a downstream transcriptional target of MYCN in neuroblastoma, whose expression is highly predictive of outcome in this disease [91, 94, 95]. The expression of another gene that is also a member of the $\mathrm{ABC}$ family of transporters, MRP4 (or $A B C C 4$ ), has also been demonstrated to be positively correlated to $M Y C N$ expression in neuroblastoma and like $M R P 1$, its over-expression is a prognostic indicator of neuroblastoma outcome [95, 96]. In fact, it has recently been shown that MYCN can coordinate the transcription of a large set of $A B C$ genes, and the expression profiles of these genes correlate with MYCN function [48].

\section{MYCN tumorigenesis}

The evidence for a clinical role of $\mathrm{MYCN}$ in the tumorigenesis of neuroblastoma was first recognised when the amplification of the $M Y C N$ oncogene was identified in 24 out of 63 primary untreated neuroblastoma tumour samples and appeared to correlate with more advanced stage of disease [97]. MYCN-amplification was subsequently associated with rapid disease progression as well as poor patient outcome in this disease [98]. Importantly, the progression-free survival of neuroblastoma patients was then shown to be dosedependent on $M Y C N$ where higher copy number resulted in lower survival. This association was independent of patient age and disease stage. $M Y C N$-amplification was later confirmed in numerous studies to be a powerful prognostic marker for predicting neuroblastoma patient outcome, independent of other clinical variables [99-102]. Determination of MYCN 
amplification status is now routinely determined in primary neuroblastomas and is one of the most powerful prognostic markers yet identified for this disease.

The MYCN oncogene is normally located on the distal short arm of chromosome 2 (2p24). This region was found to be amplified across a panel of neuroblastoma cell lines [8], and although the exact mechanism by which this occurs is unknown, the process of amplification usually results in 50 to 400 copies of the gene per cell, leading to the production of abnormally high levels of MYCN RNA and protein, presumably conferring a selective advantage to the tumour cell [103].

The potent transforming ability of MYCN has been demonstrated by several studies, while MYCN transfection studies have demonstrated that the oncoprotein plays a crucial role in neuroblastoma progression [104, 105]. Conditional overexpression of MYCN in neuroblastoma cell lines was shown to dramatically increase the growth rates and metastatic ability of these tumour cells, increase DNA synthesis, and inhibit exit from the cell cycle and neuronal differentiation [106, 107]. Furthermore, targeted expression of the MYCN oncogene in neuroectodermal cells of transgenic mice resulted in the development of neuroblastoma [3]. In these animals, human $M Y C N(h M Y C N)$ oncogene expression was targeted to neural crest cells via an upstream rat tyrosine hydroxylase promoter. Tyrosine hydroxylase is the first and rate-limiting step in catecholamine synthesis. In contrast, reduction in the MYCN RNA levels via introduction of $M Y C N$ antisense oligonucleotides in vitro as well as in vivo led to reduced rates of growth and of tumorigenicity [4, 108, 109].

Whilst MYCN-amplification has been shown to be associated with a highly malignant neuroblastoma phenotype, the precise role of this oncogene in non-amplified tumours remains controversial. Approximately $40 \%$ of those neuroblastomas that lack $M Y C N-$ amplification are nevertheless still clinically aggressive, and the clinical significance of $M Y C N$ expression in the absence of $M Y C N$-amplification, remains elusive with evidence both for and against an association with adverse outcome [110, 111]. One study that analysed both MYCN mRNA and protein levels in a cohort of non-amplified tumours, found no prognostic significance attributable to expression of this oncogene [110]. Rather, since the survival rates for older children with or without high MYCN expression were poor, the results suggested that additional factors contribute to tumour aggressiveness in this subgroup. Furthermore, in a more recent study involving 91 neuroblastoma patients, high MYCN expression was found to be associated with a favourable outcome in neuroblastomas lacking $M Y C N$-amplification [111]. Interestingly, in this study, the forced expression of MYCN significantly suppressed growth of non-amplified neuroblastoma cells by inducing apoptosis. It is possible that the prognostic value of MYCN gene expression in neuroblastoma may be an artefact of the different biology of neuroblastoma in infants compared to older children, and further well-controlled, large cohort studies will be needed in order to clarify the precise role of MYCN in non-amplified neuroblastoma.

Although the majority of the literature investigating $M Y C N$ in cancer comes from studies on neuroblastoma, this oncogene has also been shown to play a role in the tumorigenesis of other cancers, both adult and paediatric. For example, MYCN amplification and/or over- 
expression has been observed in high grade C5 serous ovarian tumours, small cell lung cancer, rhabdomyosarcoma and neuroendocrine prostate cancer [112-115], while gain of $2 p$ (and MYCN) plays a role in chronic lymphocytic leukaemia [116]. In childhood medulloblastoma, MYCN, c-MYC, and to a lesser extent MYCL, appear to be involved in the biology of this disease [117]. MYCN amplification occurs in up to $10 \%$ of medulloblastoma patients and is associated with poor clinical outcome, and like neuroblastoma, the risk of death increases with increasing copy number [117]. Furthermore, MYCN expression was found to be high in foetal cerebella, with the levels decreasing to almost absent in adult cerebella, suggesting that MYCN is essential to normal foetal development [118]. Interestingly, in this study, MYCN expression was absent from the medulloblastoma cell lines tested, which differed from the expression pattern observed in the primary tumours [118]. Finally, as with neuroblastoma, the association of MYCN mRNA levels with clinical outcome remains unclear [119] and it has been postulated that mRNA levels of both $c-M Y C$ and MYCN may only be clinically relevant in subgroups of medulloblastoma [117].

The most compelling evidence for a role of MYCN in the biology of medulloblastoma comes from two mouse models of this disease. Firstly, targeted expression of MYCN to the cerebellum in transgenic mice has demonstrated the importance of MYCN in contributing to the initiation and progression of medulloblastoma and also in the metastatic spread of disease to the spinal and paraspinal tissues via cerebral spinal fluid. Furthermore, the MYCN downstream targets Odc1, MDM2 and Fb1 were upregulated and correlated with MYCN mRNA levels [118]. The second model used targeted Smoothened (SmoA1) to the cerebella of transgenic mice, which were then crossed with mice harbouring conditional knock-out of MYCN, to demonstrate that MYCN was essential for medulloblastoma tumorigenesis [120]. These two models thus serve to demonstrate the importance of MYCN in the initiation and progression of this disease.

\section{Molecular targeting of MYCN for therapeutic benefit}

Molecular targeted therapy involves targeting malignant cell growth by directly inhibiting the function of specific molecules within a cell, namely those that are responsible for driving cancer progression. Such agents aim to block or exploit various aspects of cancer biology, such as genetic instability, proliferative signal transduction, aberrant cell cycle control, deregulated survival, angiogenesis and metastasis [121]. Numerous methods of molecular targeted therapy have been investigated, including antisense oligonucleotides (ASOs) that hybridise to and inhibit the mRNA of a specific gene; peptide nucleic acids (PNAs), which are DNA analogues that specifically hybridise to DNA and/or RNA in a complementary manner to inhibit transcription/translation of a target gene; and small interfering RNA (siRNA), which silences gene expression by inducing the sequence specific degradation of complementary mRNA or by inhibiting translation [122]. However, such technologies although useful in the laboratory, have had limited success in the clinic due to problems associated with their delivery.

Immunotherapy has also generated interest, and utilises the body's immune system to target and remove cancer cells by the recognition of certain molecular markers, or block specific 
cell receptor pathways. Another approach to molecular targeting, involves the development of synthetic small molecule inhibitors which potentially have the ability to interfere with a molecular target at multiple levels [122]. These small molecules may diffuse into cells to act directly on intracellular targets, such as inhibiting the expression of a target gene at the transcriptional or translational level, or inhibiting the function of a protein by directly binding to the protein and inducing conformational changes that prevent its interaction with other factors [123]. Synthetic small molecules are generally defined by a molecular weight cut-off of $<500 \mathrm{Da}$. They are favoured by the pharmaceutical industry because of their attractive pharmacokinetic properties, especially tumour cell penetration, and their relative ease of development and pharmaceutical production [123]. At present, strategies to develop novel small molecule inhibitors as viable therapies are aimed at using these technologies in combination with other cytotoxic drugs, with the hope of reducing drug dosages, and thus overcoming drug resistance associated with intensive chemotherapy, and reducing drugrelated toxicity and side effects.

A number of molecular mechanisms have been identified as possible targets for the treatment of neuroblastoma. However, the prominent deregulated expression and amplification of MYCN suggests that this oncogene represents an ideal target for therapeutic inhibition [124]. In addition, normal MYCN expression is restricted to the early stages of embryonic development and is virtually undetectable in normal post-natal tissues, therefore weighing in its favour as a target for inhibition. Inhibition of $M Y C N$ expression by antisense treatment against MYCN mRNA or by retinoic acid has been demonstrated to decrease proliferation and induce neuronal differentiation in neuroblastoma cells [125-127]. Furthermore, the introduction of MYCN antisense oligonucleotides in the human MYCN $(h M Y C N)$ trangenic mouse model led to reduced rates of tumour growth in these animals [4, 108, 109].

Inhibition of MYCN protein through its protein-protein interactions and protein-DNA interactions was previously seen as too difficult to target by small molecules [128]. However, it has been reported that small-molecule antagonists of MYC/MAX dimerisation interfered with c-MYC-induced oncogenic transformation of chicken embryo fibroblasts in vitro [129]. In addition, a number of endogenous MYCN/MAX antagonists such as MAX/MAX have been found to compete for binding to E-box sequences and repress transcription [130], causing cell cycle arrest, terminal differentiation or apoptosis. More recently, inhibition of c-MYC transcription via a Bromodomain and Extra Terminal Domain (BET) inhibitor, JQ1, has been described [131]. This inhibitor has been shown to disrupt cMYC mRNA synthesis by preventing the recruitment of coactivator proteins required for cMYC transcriptional initiation and mRNA elongation [131]. Furthermore, this molecule was able to decrease the tumour burden in an orthotopic mouse model of multiple myeloma. Treatment of several MYCN-amplified neuroblastoma cell lines with JQ1, resulted in a decrease in MYCN expression, although this effect was far less dramatic that that observed in a c-MYC driven cell line [132]. Despite promising evidence for targeting MYCN as a therapeutic strategy, no MYC or MYCN inhibitors have yet entered clinical trial, and further studies are required to develop effective MYCN inhibitors. 


\section{Future perspectives}

The validity for targeting MYCN for therapeutic benefit relies on the gross transforming ability of this transcription factor. MYCN represents a particularly attractive target due to its lack of expression in adult and normal paediatric tissues. Although MYCN, and MYC proteins in general are commonly viewed as "undruggable" due to the nature of these proteins, MYCN offers potential advantages at a number of levels for therapeutic inhibition, either upstream, or downstream along the MYCN transcriptional pathway. If clinically useful MYCN inhibitors can be successfully developed, they are likely to find application in combination therapies involving conventional chemotherapeutic drugs and be used as an improved approach to target aggressive cancers that are driven by this oncoprotein.

\section{Author details}

Leanna Cheung, Jayne E. Murray, Michelle Haber and Murray D. Norris Children's Cancer Institute Australia for Medical Research, Lowy Cancer Research Centre, UNSW, Sydney, Australia

\section{References}

[1] Cowling, V.H. and M.D. Cole, Mechanism of transcriptional activation by the Myc oncoproteins. Semin Cancer Biol, 2006. 16(4): p. 242-52.

[2] Grimmer, M.R. and W.A. Weiss, Childhood tumors of the nervous system as disorders of normal development. Curr Opin Pediatr, 2006. 18(6): p. 634-8.

[3] Weiss, W.A., et al., Targeted expression of MYCN causes neuroblastoma in transgenic mice. EMBO J, 1997. 16(11): p. 2985-95.

[4] Burkhart, C.A., et al., Effects of MYCN antisense oligonucleotide administration on tumorigenesis in a murine model of neuroblastoma. J Natl Cancer Inst, 2003. 95(18): p. 1394-403.

[5] Varmus, H.E., The molecular genetics of cellular oncogenes. Annu Rev Genet, 1984. 18: p. 553-612.

[6] Hayward, W.S., B.G. Neel, and S.M. Astrin, Activation of a cellular onc gene by promoter insertion in ALV-induced lymphoid leukosis. Nature, 1981. 290(5806): p. 475-80.

[7] Payne, G.S., J.M. Bishop, and H.E. Varmus, Multiple arrangements of viral DNA and an activated host oncogene in bursal lymphomas. Nature, 1982. 295(5846): p. 209-14.

[8] Schwab, M., et al., Amplified DNA with limited homology to myc cellular oncogene is shared by human neuroblastoma cell lines and a neuroblastoma tumour. Nature, 1983. 305(5931): p. 245-8.

[9] Schwab, M., MYCN in neuronal tumours. Cancer Lett, 2004. 204(2): p. 179-87.

[10] Yaylim-Eraltan, I., et al., L-myc gene polymorphism and risk of thyroid cancer. Exp Oncol, 2008. 30(2): p. 117-20.

[11] Kumimoto, H., et al., L-myc genotype is associated with different susceptibility to lung cancer in smokers. Jpn J Cancer Res, 2002. 93(1): p. 1-5.

[12] Nau, M.M., et al., L-myc, a new myc-related gene amplified and expressed in human small cell lung cancer. Nature, 1985. 318(6041): p. 69-73. 
[13] Adhikary, S. and M. Eilers, Transcriptional regulation and transformation by Myc proteins. Nat Rev Mol Cell Biol, 2005. 6(8): p. 635-45.

[14] Meyer, N. and L.Z. Penn, Reflecting on 25 years with MYC. Nat Rev Cancer, 2008. 8(12): p. 976-90.

[15] Pelengaris, S., M. Khan, and G.I. Evan, Suppression of Myc-induced apoptosis in beta cells exposes multiple oncogenic properties of Myc and triggers carcinogenic progression. Cell, 2002. 109(3): p. 321-34.

[16] Marcu, K.B., S.A. Bossone, and A.J. Patel, myc function and regulation. Annu Rev Biochem, 1992. 61: p. 809-60.

[17] Cole, M.D. and V.H. Cowling, Transcription-independent functions of MYC: regulation of translation and DNA replication. Nat Rev Mol Cell Biol, 2008. 9(10): p. 810-5.

[18] Ramsay, G., et al., Human proto-oncogene N-myc encodes nuclear proteins that bind DNA. Mol Cell Biol, 1986. 6(12): p. 4450-7.

[19] Hamann, U., et al., The MYCN protein of human neuroblastoma cells is phosphorylated by casein kinase II in the central region and at serine 367. Oncogene, 1991. 6(10): p. 1745-51.

[20] Vervoorts, J., J. Luscher-Firzlaff, and B. Luscher, The ins and outs of MYC regulation by posttranslational mechanisms. J Biol Chem, 2006. 281(46): p. 34725-9.

[21] Blackwood, E.M. and R.N. Eisenman, Max: a helix-loop-helix zipper protein that forms a sequence-specific DNA-binding complex with Myc. Science, 1991. 251(4998): p. 1211-7.

[22] Wenzel, A., et al., The N-Myc oncoprotein is associated in vivo with the phosphoprotein Max(p20/22) in human neuroblastoma cells. EMBO J, 1991. 10(12): p. 3703-12.

[23] Sakamuro, D. and G.C. Prendergast, New Myc-interacting proteins: a second Myc network emerges. Oncogene, 1999. 18(19): p. 2942-54.

[24] Bannasch, D., I. Weis, and M. Schwab, Nmi protein interacts with regions that differ between MycN and Myc and is localized in the cytoplasm of neuroblastoma cells in contrast to nuclear MycN. Oncogene, 1999. 18(48): p. 6810-7.

[25] Wood, M.A., S.B. McMahon, and M.D. Cole, An ATPase/helicase complex is an essential cofactor for oncogenic transformation by c-Myc. Mol Cell, 2000. 5(2): p. 321-30.

[26] Bannasch, D., B. Madge, and M. Schwab, Functional interaction of Yaf2 with the central region of MycN. Oncogene, 2001. 20(41): p. 5913-9.

[27] Herbst, A., et al., A conserved element in Myc that negatively regulates its proapoptotic activity. EMBO Rep, 2005. 6(2): p. 177-83.

[28] Cowling, V.H., et al., A conserved Myc protein domain, MBIV, regulates DNA binding, apoptosis, transformation, and G2 arrest. Mol Cell Biol, 2006. 26(11): p. 4226-39.

[29] Cotterman, R., et al., N-Myc regulates a widespread euchromatic program in the human genome partially independent of its role as a classical transcription factor. Cancer Res, 2008. 68(23): p. 9654-62.

[30] Knoepfler, P.S., Myc goes global: new tricks for an old oncogene. Cancer Res, 2007. 67(11): p. 5061-3.

[31] Cowling, V.H. and M.D. Cole, The Myc transactivation domain promotes global phosphorylation of the RNA polymerase II carboxy-terminal domain independently of direct DNA binding. Mol Cell Biol, 2007. 27(6): p. 2059-73.

[32] Dominguez-Sola, D., et al., Non-transcriptional control of DNA replication by c-Myc. Nature, 2007. 448(7152): p. 445-51. 
[33] McMahon, S.B., M.A. Wood, and M.D. Cole, The essential cofactor TRRAP recruits the histone acetyltransferase hGCN5 to c-Myc. Mol Cell Biol, 2000. 20(2): p. 556-62.

[34] Strahl, B.D. and C.D. Allis, The language of covalent histone modifications. Nature, 2000. 403(6765): p. 41-5.

[35] Ikura, T., et al., Involvement of the TIP60 histone acetylase complex in DNA repair and apoptosis. Cell, 2000. 102(4): p. 463-73.

[36] Frank, S.R., et al., Binding of c-Myc to chromatin mediates mitogen-induced acetylation of histone H4 and gene activation. Genes Dev, 2001. 15(16): p. 2069-82.

[37] Shen, X., et al., A chromatin remodelling complex involved in transcription and DNA processing. Nature, 2000. 406(6795): p. 541-4.

[38] Eberhardy, S.R. and P.J. Farnham, Myc recruits P-TEFb to mediate the final step in the transcriptional activation of the cad promoter. J Biol Chem, 2002. 277(42): p. 40156-62.

[39] Marhin, W.W., et al., Myc represses the growth arrest gene gadd45. Oncogene, 1997. 14(23): p. 2825-34.

[40] Wu, S., et al., Myc represses differentiation-induced p21CIP1 expression via Miz-1-dependent interaction with the $p 21$ core promoter. Oncogene, 2003. 22(3): p. 351-60.

[41] Judware, R. and L.A. Culp, Over-expression of transfected N-myc oncogene in human SKNSH neuroblastoma cells down-regulates expression of beta 1 integrin subunit. Oncogene, 1995. 11(12): p. 2599-607.

[42] Cleveland, J.L., et al., Negative regulation of c-myc transcription involves myc family proteins. Oncogene Res, 1988. 3(4): p. 357-75.

[43] Grignani, F., et al., Negative autoregulation of c-myc gene expression is inactivated in transformed cells. EMBO J, 1990. 9(12): p. 3913-22.

[44] Bell, E., et al., MYCN oncoprotein targets and their therapeutic potential. Cancer Lett, 2010. 293(2): p. 144-57.

[45] Liu, T., et al., Activation of tissue transglutaminase transcription by histone deacetylase inhibition as a therapeutic approach for Myc oncogenesis. Proc Natl Acad Sci U S A, 2007. 104(47): p. 18682-7.

[46] Tucholski, J., M. Lesort, and G.V. Johnson, Tissue transglutaminase is essential for neurite outgrowth in human neuroblastoma SH-SY5Y cells. Neuroscience, 2001. 102(2): p. 481-91.

[47] Zeng, H., et al., Transport of amphipathic anions by human multidrug resistance protein 3. Cancer Res, 2000. 60(17): p. 4779-84.

[48] Porro, A., et al., Direct and coordinate regulation of ATP-binding cassette transporter genes by Myc factors generates specific transcription signatures that significantly affect the chemoresistance phenotype of cancer cells. J Biol Chem, 2010. 285(25): p. 19532-43.

[49] Paumi, C.M., et al., Multidrug resistance protein (MRP) 1 and MRP3 attenuate cytotoxic and transactivating effects of the cyclopentenone prostaglandin, 15-deoxy-Delta(12,14)prostaglandin J2 in MCF7 breast cancer cells. Biochemistry, 2003. 42(18): p. 5429-37.

[50] Hatzi, E., et al., N-myc oncogene overexpression down-regulates IL-6; evidence that IL-6 inhibits angiogenesis and suppresses neuroblastoma tumor growth. Oncogene, 2002. 21(22): p. 3552-61.

[51] Schneider, A., et al., Association of Myc with the zinc-finger protein Miz-1 defines a novel pathway for gene regulation by Myc. Curr Top Microbiol Immunol, 1997. 224: p. 137-46.

[52] Peukert, K., et al., An alternative pathway for gene regulation by Myc. EMBO J, 1997. 16(18): p. $5672-86$. 
[53] Ikegaki, N., et al., De novo identification of MIZ-1 (ZBTB17) encoding a MYC-interacting zinc-finger protein as a new favorable neuroblastoma gene. Clin Cancer Res, 2007. 13(20): p. 6001-9.

[54] Wanzel, M., S. Herold, and M. Eilers, Transcriptional repression by Myc. Trends Cell Biol, 2003. 13(3): p. 146-50.

[55] Brenner, C., et al., Myc represses transcription through recruitment of DNA methyltransferase corepressor. EMBO J, 2005. 24(2): p. 336-46.

[56] Schwab, M., Human neuroblastoma: from basic science to clinical debut of cellular oncogenes. Naturwissenschaften, 1999. 86(2): p. 71-8.

[57] Laird-Offringa, I.A., What determines the instability of c-myc proto-oncogene mRNA? Bioessays, 1992. 14(2): p. 119-24.

[58] Mirsky, R., et al., Novel signals controlling embryonic Schwann cell development, myelination and dedifferentiation. J Peripher Nerv Syst, 2008. 13(2): p. 122-35.

[59] Stanton, B.R., et al., Loss of $N$-myc function results in embryonic lethality and failure of the epithelial component of the embryo to develop. Genes Dev, 1992. 6(12A): p. 2235-47.

[60] Strieder, V. and W. Lutz, E2F proteins regulate MYCN expression in neuroblastomas. J Biol Chem, 2003. 278(5): p. 2983-9.

[61] Morrow, M.A., et al., Interleukin-7 induces $N$-myc and c-myc expression in normal precursor B lymphocytes. Genes Dev, 1992. 6(1): p. 61-70.

[62] Wada, R.K., et al., Cell type-specific expression and negative regulation by retinoic acid of the human N-myc promoter in neuroblastoma cells. Oncogene, 1992. 7(4): p. 711-7.

[63] Bentley, D.L. and M. Groudine, A block to elongation is largely responsible for decreased transcription of c-myc in differentiated HL60 cells. Nature, 1986. 321(6071): p. 702-6.

[64] Sivak, L.E., et al., A novel intron element operates posttranscriptionally To regulate human Nmyc expression. Mol Cell Biol, 1999. 19(1): p. 155-63.

[65] Keene, R.G., et al., Transcriptional pause, arrest and termination sites for RNA polymerase II in mammalian $\mathrm{N}$ - and c-myc genes. Nucleic Acids Res, 1999. 27(15): p. 3173-82.

[66] Xu, L., S.D. Morgenbesser, and R.A. DePinho, Complex transcriptional regulation of myc family gene expression in the developing mouse brain and liver. Mol Cell Biol, 1991. 11(12): p. 6007-15.

[67] Brewer, G. and J. Ross, Poly (A) shortening and degradation of the 3'A+U-rich sequences of human c-myc mRNA in a cell-free system. Mol Cell Biol, 1988. 8(4): p. 1697-708.

[68] Ross, J., mRNA stability in mammalian cells. Microbiol Rev, 1995. 59(3): p. 423-50.

[69] Bernstein, P.L., et al., Control of $c-m y c m R N A$ half-life in vitro by a protein capable of binding to a coding region stability determinant. Genes Dev, 1992. 6(4): p. 642-54.

[70] Chagnovich, D. and S.L. Cohn, Activity of a $40 \mathrm{kDa}$ RNA-binding protein correlates with MYCN and c-fos mRNA stability in human neuroblastoma. Eur J Cancer, 1997. 33(12): p. 2064-7.

[71] Jopling, C.L. and A.E. Willis, N-myc translation is initiated via an internal ribosome entry segment that displays enhanced activity in neuronal cells. Oncogene, 2001. 20(21): p. 2664-70.

[72] Sjostrom, S.K., et al., The Cdk1 complex plays a prime role in regulating $N$-myc phosphorylation and turnover in neural precursors. Dev Cell, 2005. 9(3): p. 327-38.

[73] Knoepfler, P.S., P.F. Cheng, and R.N. Eisenman, N-myc is essential during neurogenesis for the rapid expansion of progenitor cell populations and the inhibition of neuronal differentiation. Genes Dev, 2002. 16(20): p. 2699-712. 
[74] Kim, H.T., et al., Certain pairs of ubiquitin-conjugating enzymes (E2s) and ubiquitin-protein ligases (E3s) synthesize nondegradable forked ubiquitin chains containing all possible isopeptide linkages. J Biol Chem, 2007. 282(24): p. 17375-86.

[75] Eilers, M., S. Schirm, and J.M. Bishop, The MYC protein activates transcription of the alphaprothymosin gene. EMBO J, 1991. 10(1): p. 133-41.

[76] Bello-Fernandez, C., G. Packham, and J.L. Cleveland, The ornithine decarboxylase gene is a transcriptional target of c-Myc. Proc Natl Acad Sci U S A, 1993. 90(16): p. 7804-8.

[77] Grandori, C. and R.N. Eisenman, Myc target genes. Trends Biochem Sci, 1997. 22(5): p. 177-81.

[78] Cole, M.D. and S.B. McMahon, The Myc oncoprotein: a critical evaluation of transactivation and target gene regulation. Oncogene, 1999. 18(19): p. 2916-24.

[79] Zeller, K.I., et al., An integrated database of genes responsive to the Myc oncogenic transcription factor: identification of direct genomic targets. Genome Biol, 2003. 4(10): p. R69.

[80] Guccione, E., et al., Myc-binding-site recognition in the human genome is determined by chromatin context. Nat Cell Biol, 2006. 8(7): p. 764-70.

[81] McMahon, S.B., Control of nucleotide biosynthesis by the MYC oncoprotein. Cell Cycle, 2008. 7(15): p. 2275-6.

[82] Bell, E., J. Lunec, and D.A. Tweddle, Cell cycle regulation targets of MYCN identified by gene expression microarrays. Cell Cycle, 2007. 6(10): p. 1249-56.

[83] Lastowska, M., et al., Identification of candidate genes involved in neuroblastoma progression by combining genomic and expression microarrays with survival data. Oncogene, 2007. 26(53): p. $7432-44$.

[84] Dang, C.V., et al., The c-Myc target gene network. Semin Cancer Biol, 2006. 16(4): p. 253-64.

[85] Nesbit, C.E., J.M. Tersak, and E.V. Prochownik, MYC oncogenes and human neoplastic disease. Oncogene, 1999. 18(19): p. 3004-16.

[86] Slack, A., G. Lozano, and J.M. Shohet, MDM2 as MYCN transcriptional target: implications for neuroblastoma pathogenesis. Cancer Lett, 2005. 228(1-2): p. 21-7.

[87] Fan, J., et al., Time-dependent c-Myc transactomes mapped by Array-based nuclear run-on reveal transcriptional modules in human B cells. PLoS One, 2010. 5(3): p. e9691.

[88] Pegg, A.E., Polyamine metabolism and its importance in neoplastic growth and a target for chemotherapy. Cancer Res, 1988. 48(4): p. 759-74.

[89] Hogarty, M.D., et al., ODC1 is a critical determinant of MYCN oncogenesis and a therapeutic target in neuroblastoma. Cancer Res, 2008. 68(23): p. 9735-45.

[90] Bordow, S.B., et al., Expression of the multidrug resistance-associated protein (MRP) gene correlates with amplification and overexpression of the N-myc oncogene in childhood neuroblastoma. Cancer Res, 1994. 54(19): p. 5036-40.

[91] Norris, M.D., et al., Expression of the gene for multidrug-resistance-associated protein and outcome in patients with neuroblastoma. N Engl J Med, 1996. 334(4): p. 231-8.

[92] Haber, M., et al., Altered expression of the MYCN oncogene modulates MRP gene expression and response to cytotoxic drugs in neuroblastoma cells. Oncogene, 1999. 18(17): p. 2777-82.

[93] Munoz, M., et al., Role of the MRP1/ABCC1 multidrug transporter protein in cancer. IUBMB Life, 2007. 59(12): p. 752-7.

[94] Manohar, C.F., et al., MYCN-mediated regulation of the MRP1 promoter in human neuroblastoma. Oncogene, 2004. 23(3): p. 753-62. 
[95] Henderson, M.J., et al., ABCC multidrug transporters in childhood neuroblastoma: clinical and biological effects independent of cytotoxic drug efflux. J Natl Cancer Inst, 2011. 103(16): p. 1236-51.

[96] Norris, M.D., et al., Expression of multidrug transporter MRP4/ABCC4 is a marker of poor prognosis in neuroblastoma and confers resistance to irinotecan in vitro. Mol Cancer Ther, 2005. 4(4): p. 547-53.

[97] Brodeur, G.M., et al., Amplification of N-myc in untreated human neuroblastomas correlates with advanced disease stage. Science, 1984. 224(4653): p. 1121-4.

[98] Seeger, R.C., et al., Association of multiple copies of the N-myc oncogene with rapid progression of neuroblastomas. N Engl J Med, 1985. 313(18): p. 1111-6.

[99] Bartram, C.R. and F. Berthold, Amplification and expression of the N-myc gene in neuroblastoma. Eur J Pediatr, 1987. 146(2): p. 162-5.

[100] Nakagawara, A., et al., Amplification of N-myc oncogene in stage II and IVS neuroblastomas may be a prognostic indicator. J Pediatr Surg, 1987. 22(5): p. 415-8.

[101] Brodeur, G.M., et al., International criteria for diagnosis, staging, and response to treatment in patients with neuroblastoma. J Clin Oncol, 1988. 6(12): p. 1874-81.

[102] Brodeur, G.M. and C.T. Fong, Molecular biology and genetics of human neuroblastoma. Cancer Genet Cytogenet, 1989. 41(2): p. 153-74.

[103] Amler, L.C. and M. Schwab, Multiple amplicons of discrete sizes encompassing N-myc in neuroblastoma cells evolve through differential recombination from a large precursor DNA. Oncogene, 1992. 7(4): p. 807-9.

[104] Brodeur, G.M., Neuroblastoma: clinical significance of genetic abnormalities. Cancer Surv, 1990. 9(4): p. 673-88.

[105] Xu, L., et al., Loss of transcriptional attenuation in N-myc is associated with progression towards a more malignant phenotype. Oncogene, 1995. 11(9): p. 1865-72.

[106] Bogenmann, E., M. Torres, and H. Matsushima, Constitutive N-myc gene expression inhibits trkA mediated neuronal differentiation. Oncogene, 1995. 10(10): p. 1915-25.

[107] Lutz, W., et al., Conditional expression of N-myc in human neuroblastoma cells increases expression of alpha-prothymosin and ornithine decarboxylase and accelerates progression into $S$ phase early after mitogenic stimulation of quiescent cells. Oncogene, 1996. 13(4): p. 803-12.

[108] Whitesell, L., A. Rosolen, and L.M. Neckers, Antisense suppression of N-myc expression inhibits the transdifferentiation of neuroectoderm tumor cell lines. Prog Clin Biol Res, 1991. 366: p. 45-54.

[109] Whitesell, L., A. Rosolen, and L.M. Neckers, In vivo modulation of N-myc expression by continuous perfusion with an antisense oligonucleotide. Antisense Res Dev, 1991. 1(4): p. 343-50.

[110] Cohn, S.L., et al., MYCN expression is not prognostic of adverse outcome in advanced-stage neuroblastoma with nonamplified MYCN. J Clin Oncol, 2000. 18(21): p. 3604-13.

[111] Tang, X.X., et al., The MYCN enigma: significance of MYCN expression in neuroblastoma. Cancer Res, 2006. 66(5): p. 2826-33.

[112] Tonelli, R., et al., Antitumor activity of sustained N-myc reduction in rhabdomyosarcomas and transcriptional block by antigene therapy. Clin Cancer Res, 2012. 18(3): p. 796-807.

[113] Helland, A., et al., Deregulation of MYCN, LIN28B and LET7 in a molecular subtype of aggressive high-grade serous ovarian cancers. PLoS One, 2011. 6(4): p. e18064. 
[114] Kim, Y.H., et al., Combined microarray analysis of small cell lung cancer reveals altered apoptotic balance and distinct expression signatures of MYC family gene amplification. Oncogene, 2006. 25(1): p. 130-8.

[115] Beltran, H., et al., Molecular Characterization of Neuroendocrine Prostate Cancer and Identification of New Drug Targets. Cancer Discov, 2011. 1(6): p. 487-495.

[116] Ma, D., et al., Array Comparative Genomic Hybridization Analysis Identifies Recurrent Gain of Chromosome 2p25.3 Involving the ACP1 and MYCN Genes in Chronic Lymphocytic Leukemia. Clinical Lymphoma Myeloma and Leukemia, 2011. 11, Supplement 1(0): p. S17-S24.

[117] Ryan, S.L., et al., MYC family amplification and clinical risk-factors interact to predict an extremely poor prognosis in childhood medulloblastoma. Acta Neuropathol, 2012. 123(4): p. 501-13.

[118] Swartling, F.J., et al., Pleiotropic role for MYCN in medulloblastoma. Genes Dev, 2010. 24(10): p. 1059-72.

[119] Eberhart, C.G., et al., Histopathological and molecular prognostic markers in medulloblastoma: c-myc, N-myc, TrkC, and anaplasia. J Neuropathol Exp Neurol, 2004. 63(5): p. 441-9.

[120] Hatton, B.A., et al., N-myc is an essential downstream effector of Shh signaling during both normal and neoplastic cerebellar growth. Cancer Res, 2006. 66(17): p. 8655-61.

[121] Garrett, M.D. and P. Workman, Discovering novel chemotherapeutic drugs for the third millennium. Eur J Cancer, 1999. 35(14): p. 2010-30.

[122] Vita, M. and M. Henriksson, The Myc oncoprotein as a therapeutic target for human cancer. Semin Cancer Biol, 2006. 16(4): p. 318-30.

[123] Jain, R.K., Delivery of novel therapeutic agents in tumors: physiological barriers and strategies. J Natl Cancer Inst, 1989. 81(8): p. 570-6.

[124] Lu, X., A. Pearson, and J. Lunec, The MYCN oncoprotein as a drug development target. Cancer Lett, 2003. 197(1-2): p. 125-30.

[125] Negroni, A., et al., Decrease of proliferation rate and induction of differentiation by a MYCN antisense DNA oligomer in a human neuroblastoma cell line. Cell Growth Differ, 1991. 2(10): p. 511-8.

[126] Marshall, G.M., et al., Increased retinoic acid receptor gamma expression suppresses the malignant phenotype and alters the differentiation potential of human neuroblastoma cells. Oncogene, 1995. 11(3): p. 485-91.

[127] Thiele, C.J., C.P. Reynolds, and M.A. Israel, Decreased expression of N-myc precedes retinoic acid-induced morphological differentiation of human neuroblastoma. Nature, 1985. 313(6001): p. 404-6.

[128] Soucek, L., et al., Modelling Myc inhibition as a cancer therapy. Nature, 2008. 455(7213): p. 679-83.

[129] Berg, T., et al., Small-molecule antagonists of Myc/Max dimerization inhibit Myc-induced transformation of chicken embryo fibroblasts. Proc Natl Acad Sci U S A, 2002. 99(6): p. 3830-5.

[130] Facchini, L.M. and L.Z. Penn, The molecular role of Myc in growth and transformation: recent discoveries lead to new insights. FASEB J, 1998. 12(9): p. 633-51.

[131] Delmore, J.E., et al., BET bromodomain inhibition as a therapeutic strategy to target c-Myc. Cell, 2011. 146(6): p. 904-17.

[132] Mertz, J.A., et al., Targeting MYC dependence in cancer by inhibiting BET bromodomains. Proc Natl Acad Sci U S A, 2011. 108(40): p. 16669-74. 